\title{
BMC Health Services Research reviewer acknowledgement 2014
}

\section{Christopher Morrey}

Perri 6

UK

Jos Aarts

Netherlands

Karina Aase

Norway

John Abebrese Boateng

Ghana

Seye Abimbola

Nigeria

Mauro Henrique Abreu

Brazil

Amani Abu- Shaheen

Saudi Arabia

Monica Acevedo

Chile

Ezekiel Taiwo Adebayo

Nigeria

Adedeji Adekanye

Nigeria

Marian Ådnanes

Norway

Martin Nyaaba Adokiya

Ghana

Willem Aelvoet

Belgium

Basema Afram

Netherlands

Bushra Afroze

Pakistan
Girdhar Agarwal

India

Sohail Agha

USA

Peter Agyei-Baffour

Ghana

Mohamed Azmi Ahmad Hassali

Malaysia

Maryam Ahmadian

Malaysia

Rukhsana Ahmed

Indonesia

Aima Ahonkhai

USA

Moses Aikins

Ghana

Bolajoko Ajoke Aina

Nigeria

James Akazili

Ghana

Halida Akhter

Bangladesh

Shahriar Akter

Australia

Patricia Akweongo

Ghana

Fadi Al Zoubi

Canada

Mohammed Alam

UK
Monira Alarouj

Kuwait

Tit Albreht

Slovenia

Vassilis Aletras

Greece

Yasmin Alhafaji

Netherlands

Syed Aljunid

Malaysia

Alejandro Allepuz

Spain

Sara Allin

Canada

Pascale Allotey

Malaysia

Helen Alm

Sweden

Carlos Alvarez

USA

Emmanuel Ameh

Nigeria

Joshua Amo-Adjei

Ghana

Seyi Ladele Amosun

South Africa

Eugenia Amporfu

Ghana

Elizabeth Andersen

Canada

Correspondence: christopher.morrey@biomedcentral.com

BioMed Central, Floor 6, 236 Gray's Inn Road, WC1X 8HB London, United

Kingdom 
Henning Boje Andersen

Denmark

Allen Anderson
USA
Kurt Anderson
USA
Philippa Anderson
USA

Ruth A. Anderson

USA

Vasanth Andrews

UK

Federica Angeli

Netherlands

Salvatore Annunziata

Italy

Rose Anorlu

Nigeria

Zahid Ansari

Australia

Fernando Antonanzas

Spain

Tony Antoniou

Canada

Jose Leopoldo Ferreira Antunes

Brazil

Konstantinos Antypas

Norway

Belkis Aracena

Mexico

Carlos Arango

Colombia

Edson Araujo

USA

Laura Arbour

Canada

Norm Archer

Canada

Greg Arling

USA

Cherie Armour

UK

Greg Armstrong

Australia
Joanna Armstrong Schellenberg

UK

Britt Arrelov

Sweden

Jelena Arsenijevic

Netherlands

Genevieve Aryeetey

Ghana

Augustine Asante

Australia

Raj Ashar

USA

Toni Ashton

New Zealand

Yaregal Asres

Ethiopia

Kostas Athanasakis

Greece

Vasilios Athyros

Greece

Roger Ayimbillah Atinga

Ghana

Lukoye Atwoli

Kenya

Carolyn Audet

USA

Asa Audulv

Sweden

Hanna Augustsson

Sweden

Isabelle Aujoulat

Belgium

Ahmed Awaisu

Qatar

Japheth Awiti

Kenya

Koku Awoonor-Williams

Ghana

Baffour Awuah

Ghana

Iben Axén

Sweden

Muhammad Ayub

Pakistan
Adriana Baban

Romania

Omar Badawi

USA

Franz G. Bader

Germany

Mario Bagat

Croatia

Cheick Oumar Bagayoko

Mali

Ilaria Baiardini

Italy

Frank Baiden

Ghana

Charles Bailey

USA

Daryl Bainbridge

Canada

Petra Baji

Hungary

Solome Kiribakka Bakeera

Uganda

Roland Bal

Netherlands

Eileen Baldry

Australia

Anne Balossier

France

Lawrence Barat

USA

Anna Maria Bargagli

Italy

Lawrence Barker

USA

Paul Barnett

USA

Mauro Barni

Italy

Hugh Barr

UK

Helen Barratt

UK

Patricia Barrionuevo

Peru 
Melanie Barwick

Canada

Robert Basaza

Uganda

Caroline Bastiaenen

Netherlands

Lori Bastian

USA

Moses Bateganya

Uganda

Philip Batterham

Australia

Jennifer Baumbusch

Canada

Cynthia Baur

USA

Alia Bazzy-Asaad

USA

Davida Becker

USA

Katrien Beeckman

Belgium

Kendall Bein

Australia

Derek Bell

UK

Gill Bell

UK

Lorraine Bell

Canada

Mary Bell

Canada

Sandra Benavides

USA

Kathleen Beniuk

Canada

Paola Berchialla

Italy

Peter Berchtold

Switzerland

Sean Berenholtz

USA

Anne-Marie Bergh

South Africa
Werner Bergholz

Germany

Trine Strand Bergmo

Norway

Linda Bergofsky

USA

Julie Bernhardt

Australia

Allan Best

Canada

Kurt Bestehorn

Germany

Martie van Beuzekom

Netherlands

Ran Bhamra

UK

Upendra Bhojani

India

Rama Bhunia

India

Barbara Bien

Poland

Jean Joel Bigna

Cameroon

Ugur Bilge

Turkey

Stephen Billett

Australia

Aref Bin Abdulhak

Saudi Arabia

Sarah Birken

USA

Marie Bismark

New Zealand

Vesna Bjelic Radisic

Austria

Anne Mette Bjerkan

Norway

Wendy Bjornson

USA

Duane Blaauw

South Africa

Regis Blais

Canada
Christopher Blanchette

USA

Marc-André Blanchette

Canada

Jane Blazeby

UK

Ilse Blignault

Australia

Catherine Bliss

USA

Paul Blumenthal

USA

Jennifer Blumenthal-Barby

USA

Ian Blunt

UK

Thomas Bodenheimer

USA

Said Bodur

Turkey

J Ties Boerma

Switzerland

Peter Bogaty

Canada

Thomas Böhler

Germany

Philippe Boierling

France

Katrien Bombeke

Belgium

Gary Bond

USA

Cecile Boot

Netherlands

Josephine Borghi

UK

Sander Borgsteede

Netherlands

Mats Borjesson

Sweden

Ann Borzecki

USA

Thomas John Bossert

USA 
Michel Botbol

France

Marge Bott

USA

Alex Bottle

UK

Michel Boudreaux

USA

Mary Boulton

UK

David Bowrey

UK

Laurent Boyer

France

Jennifer Boyko

Canada

Natalie Bradford

Australia

Elizabeth Bradley

USA

Sara Bradley

USA

Zoe Brady

Australia

Kate Brain

UK

Anneke Brand

Netherlands

Caroline Brand

Australia

Aase Brandt

Denmark

Susan Bratton

USA

Karen Bremner

Canada

Patricia Brennan

USA

Daniel Bressington

UK

Hans-Peter Brezinschek

Austria

Jackie Bridges

UK
William Brieger

USA

Annette Briley

UK

Michelle Bronze

South Africa

Peter Brooks

Australia

Edward Broughton

USA

George Browman

Canada

Jamie Brown

UK

Tony Brown

USA

Natasha Brusco

Australia

Maurice Bucagu

Switzerland

Angela Buchholz

Germany

Christoph Buhrer

Germany

Alexis Bujold

Canada

Yilmaz Bulbul

Turkey

Alicia Bunger

USA

Robert Burke

USA

Greer Burkholder

USA

Christopher Burton

UK

Gerard Bury

Ireland

Maria Antonella Burza

Sweden

Erik Buskens

Netherlands

Monica Busse

UK
Silvia Bustacchini

Italy

Sandra Butler

USA

David Byfield

UK

Jens Byskov

Denmark

Liam Caffery

Australia

Lucero Cahuana-Hurtado

Mexico

Stefano Calciolari

Switzerland

Amaia Calderón-Larrañaga

Spain

Jennifer Callaghan-Koru

USA

Claire Cameron

New Zealand

John Campbell

Japan

Carlos Campillo-Artero

Spain

Maureen Canavan

USA

Kenneth Candido

USA

Sandra Capra

Australia

Massimo Cardillo

Italy

Misericordia Carles

Spain

Siw Carlfjord

Sweden

Ken Carlson

Algeria

Eloise Carr

Canada

Henry Carretta

USA

Ewart Carson

UK 


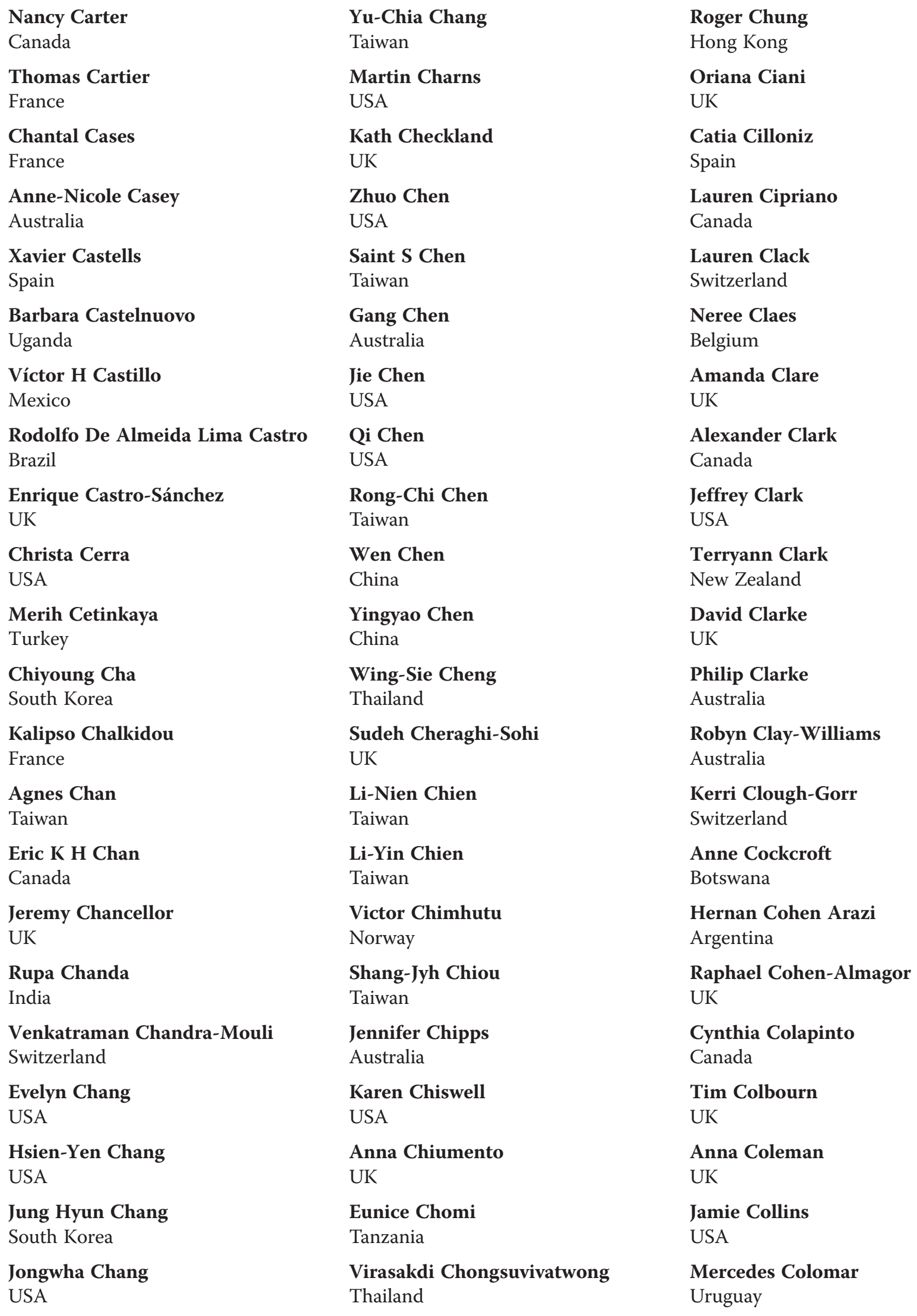


Tracy Comans

Australia

Elizabeth Comino

Australia

Anita Conforti

Italy

Richard Cook

Sweden

Evelyn Cornelissen

Canada

Tony Cornford

UK

Gloria Coronado

USA

Julieta Corral

Spain

Aline Corvol

France

Andrew Costa

Canada

Massimo Costantini

Italy

Fernando Coto

Costa Rica

Francesc Cots

Spain

Susan Cottrell

UK

Angela Coulter

UK

Simon Coulton

UK

Ian Couper

South Africa

Karen Courtney

Canada

Joseph Couto

USA

Janneke Cox

Netherlands

Peter Craig

UK

Peter Cram

Canada
Jane Murray Cramm

Netherlands

Grainne Crealey

UK

Andreea Creanga

USA

Torrey Creed

USA

Jo Crichton

UK

Bart Criel

Belgium

Clare Crowley

UK

Eduardo Cruz

Portugal

Joaquin Cubiella

Spain

Larry Culpepper

USA

Frances Cunningham

Australia

Maria Paula Curado

France

Alessandro Curto

Italy

Jonathan Cylus

UK

Carolyn Czoski Murray

UK

Matiwos Daba

Ethiopia

Katie Dainty

Canada

Gavin Daker-White

UK

David Dale

USA

Philip Ayizem Dalinjong

Ghana

Maurice Dalton

USA

Barbara Daly

New Zealand
Gianfranco Damiani

Italy

Laura Damschroder

USA

M Carolina Danovaro-Holliday

USA

Andriy Danyliv

Ukraine

Blair Darney

USA

Umakant Dash

India

Marie Dauvrin

Belgium

Larry Davidson

USA

Ruth Davies

UK

Keith Davis

USA

Peter Davis

New Zealand

Damon Daylamani-Zad

UK

Manuela De Allegri

Germany

Dinny De Bakker

Netherlands

Margaretha Christine De Bruijne

Netherlands

Ayesha De Costa

Sweden

Laure De Decker

France

Simon De Lusignan

UK

Victoria De Menil

UK

Matthieu De Stampa

France

Anniza De Villiers

South Africa

Linda De Villiers

South Africa 
G. Ardine De Wit

Netherlands

Paquita De Zulueta

UK

Jonas Debesay

Norway

Anja Declercq

Belgium

Tom Decroo

Belgium

Ryan Deforge

Canada

Ellen Tveter Deilkås

Norway

Veronique Del Marmol

Belgium

Kim Delbaere

Australia

Cristina Del-Ben

Brazil

Derek Delia

USA

Thérèse Delvaux

Benin

Nadia Demarteau

Belgium

Petra Denig

Netherlands

Mike Dent

UK

Richard Derman

USA

Jane Desborough

Australia

Domino Determann

Netherlands

Mary Devany

USA

Angela Dew

Australia

Sanket Dhruva

USA

Paula Diab

South Africa
Vakaramoko Diaby

USA

Esperanza Diaz

Norway

Hassan H. Dib

Canada

Helen Dickinson

Australia

Virginia Dickson-Swift

Australia

Marjolein Dieleman

Netherlands

Hans Jochen Diesfeld

Germany

Vance Dietz

USA

Carmen Desiree Dirksen

Netherlands

Simon Dixon

UK

Mamuka Djibuti

Georgia

Fahdi Dkhimi

Belgium

Mai Do

USA

Charles Doarn

USA

Henry Doctor

Nigeria

Carmen Dolea

Switzerland

Sara Donetto

UK

Amol Dongre

India

Claire Donnellan

Ireland

Catherine Donnelly

Canada

Caroline Donovan

Australia

Diane Doran

Canada
William Doucette

USA

Lynda Doward

UK

Bryan Dowd

USA

Dawn Dowding

USA

Julia Downing

Uganda

Therese Dowswell

UK

Colleen Doyle

Australia

Aline Drapeau

Canada

David Dror

India

Arik Drucker

Canada

Renee Du Toit

South Africa

Stephen Duckett

Canada

Antoine Duclos

France

Dmitry Dukhovny

USA

James Dunbar

Australia

Cathy Duncan

Australia

Anna Dunér

Sweden

Alison Dunkley

UK

Luis Durán-Arenas

Mexico

Angela Durey

Australia

Jo Durham

Australia

Gilles Dussault

Portugal 
Hemant Dwivedi

India

Rosamond Dwyer

Australia

Elin Dysvik

Norway

Mervyn Eadie

Australia

Phyllis Easton

UK

Jennifer E Edelman

USA

Dale Edgar

Australia

Dawn Edge

UK

Anbrasi Edward

USA

Alun Edwards

Canada

Jane Edwards

Australia

Louisa Edwards

UK

Judith Effken

USA

Mary Egan

Canada

Marlene J Egger

USA

Ashley Eggman

USA

Jan P Ehlers

Germany

Elizabeth Ekirapa-Kiracho

Uganda

Maguy El Hajj

Qatar

Ann Catrine Eldh

Sweden

Omar El-Gayar

USA

C Raina Elley

New Zealand
Martin Emmert

Germany

Liam Ennis

UK

David Epstein

Spain

Mirela Eric

Serbia

Erhan Eser

Turkey

Dominick Esposito

USA

Silvia Ess

Switzerland

Marie-Louise Essink-Bot

Netherlands

Carole A Estabrooks

Canada

Enyi Etiaba

Nigeria

Catrin Evans

UK

Roni Evans

USA

Combier Evelyne

France

Soludo Eze

Nigeria

Boniface Ikenna Eze

Nigeria

Echezona Ezeanolue USA

Ogochukwu Ezeoke

Nigeria

Nkoli Ezumah

Nigeria

Esamai Fabian

Kenya

Reza Fadayevatan

Iran

Michael Falster

Australia

Victoria Fan

USA
Mahnaz Fanaian

Australia

Pengqian Fang

China

Yu Fang

China

Folayemi Faponle

Nigeria

Jane Farmer

Australia

Saeed Farooq

UK

Maryam Farooqui

Malaysia

Karen Farris

USA

Fereshteh Farzianpour

Iran

Denise Feil

USA

Bo Feng

USA

Zhanchun Feng

China

Jennifer Fenwick

Australia

Angeline Ferdinand

Australia

Laurie Anne Ferguson

USA

Laura Ferguson

UK

Ewan Ferlie

UK

Alfio Ferlito

Italy

Mazen Saleh Ferwana

Saudi Arabia

Bolanle Fetuga

Nigeria

Guido Filler

Canada

Sally Findley

USA 
Sonja Firth

Australia

Belal Firwana

USA

Alastair Fischer

UK

Michael Fischer

USA

Ane Fisker

Denmark

Linda Fleisher

USA

Steffen Flessa

Germany

Monica Julie Fletcher

UK

Jan Florin

Sweden

Linda Fogarty

USA

Ted C T Fong

Hong Kong

Sharon Fonn

South Africa

Lindsay Forbes

UK

Jane Ford

Australia

Jay Ford

USA

Roberto Forero

Australia

Yvonne Forsell

Sweden

Louise Forsetlund

Norway

Martin Fortin

Canada

Kim Foster

Australia

Jean Christophe Fotso

Kenya

Deborah Fox

Australia
Gregory Fox

Australia

Margaret Fox

UK

Vassilis Fragoulakis

Greece

Anneke Leijntje Francke

Netherlands

Molly Franke

USA

Bryony Dean Franklin

UK

Randall Fransoo

Canada

Richard K Freeman

USA

Simon French

Canada

Atle Fretheim

Norway

Tobias Freund

Germany

Maria Freytsis

USA

Jan C Frich

Norway

Kevin Frick

USA

Roland Friele

Netherlands

Laura Fruhen

UK

Robert Fryatt

USA

Naomi Fulop

UK

Martha Funnell

USA

Kiyohide Fushimi

Japan

Anna Gagliardi

Canada

Arnaud Gagneur

Canada
Marie-Pierre Gagnon

Canada

Petros Galanis

Greece

Christopher Gale

New Zealand

Katie Gallacher

UK

James Gallagher

Ireland

Gisselle Gallego

Australia

Rebecca Ganann

Canada

David Gans

USA

Guodong Gao

USA

Jian Gao

USA

Marcial Garcia-Rojo

Spain

Karen Gardner

Australia

Allan Garland

Canada

Paul Garner

UK

James Gaughan

UK

Robin Gauld

New Zealand

Lara Gautier

France

Florian Gebreiter

UK

Alison Gee

Australia

Jo Geere

UK

Katrin Gehring

Switzerland

Alexander Geissler Germany 
Stacie Geller

USA

Becky Genberg

USA

Stephen Genuis

Canada

Pradeep Paul George

Singapore

Max Geraedts

Germany

Karen Gerard

UK

Saswata Ghosh

India

Swapnil Ghotane

UK

Francesco Gianfagna

Italy

Andry Giannakou

Cyprus

Maggie Gibson

Canada

Rod Gibson

UK

Julie Gilbert

Canada

Julia Gilmartin

UK

Sarah Gimbel

USA

Rosa Gini

Italy

Liane Ginsburg

Canada

Michael Gionfriddo

USA

Carlo Bruno Giorda

Italy

Edmond Girasek

Hungary

Stefan Gisin

Switzerland

Sophie Githinji

Kenya
Jody Hoffer Gittell

USA

Brian Godman

Sweden

Dianne Goeman

Australia

Katja Goetz

Germany

Lisa Gold

Australia

Rachel Gold

USA

Shaun Goldfinch

Fiji

David Goldsbury

Australia

Daniel Goldstein

USA

Marc Goldstein

USA

Sarah Gollust

USA

Barbara Gomes

UK

Francesc Xavier Gomez-Olive Casas

South Africa

Antonio Gómez-Outes

Spain

Eduardo Gonzalez-Pier

Mexico

María Cecilia González-Robledo

Mexico

Claire Goodman

UK

Catherine Goodman

Kenya

Yelena Gorina

USA

B L Gorissen

Netherlands

Holger Gothe

Austria

Lesley Gotlib Conn

Canada
Natasha Gous

South Africa

Pedro Gozalo

USA

Markus Grabka

Germany

Sandra Grace

Australia

Jonathan Graffy

UK

Chris Graham

UK

Gonzalo Grandes

Spain

Grazia Grazzini

Italy

Laurie Grealish

Australia

Esther Green

Canada

Dan Greenberg

Israel

William Greene

USA

David Greenfield

Australia

Trisha Greenhalgh

UK

Catherine Grenier

France

Peter Grimison

Australia

Karen Grimmer

Australia

Peter Groenewegen

Netherlands

Vigdis Abrahamsen Grøndahl

Norway

Charu Grover

Australia

Andrea Gruneir

Canada

Larry Gruppen

USA 


\begin{tabular}{|c|c|c|}
\hline $\begin{array}{l}\text { Kristina Gryboski } \\
\text { USA }\end{array}$ & $\begin{array}{l}\text { Christopher Halpin } \\
\text { USA }\end{array}$ & $\begin{array}{l}\text { Mark Harris } \\
\text { Australia }\end{array}$ \\
\hline $\begin{array}{l}\text { Enza Gucciardi } \\
\text { Canada }\end{array}$ & $\begin{array}{l}\text { Kate Halton } \\
\text { Australia }\end{array}$ & $\begin{array}{l}\text { Mark Harrison } \\
\text { UK }\end{array}$ \\
\hline $\begin{array}{l}\text { Mara Guerreiro } \\
\text { UK }\end{array}$ & $\begin{array}{l}\text { Amy Halverson } \\
\text { USA }\end{array}$ & $\begin{array}{l}\text { Michael Harrison } \\
\text { USA }\end{array}$ \\
\hline $\begin{array}{l}\text { P Cristian Gugiu } \\
\text { USA }\end{array}$ & $\begin{array}{l}\text { Syed Usman Hamdani } \\
\text { Pakistan }\end{array}$ & $\begin{array}{l}\text { Simon Harrison } \\
\text { Australia }\end{array}$ \\
\hline $\begin{array}{l}\text { Janice Gullick } \\
\text { Australia }\end{array}$ & $\begin{array}{l}\text { Robert Hamm } \\
\text { USA }\end{array}$ & $\begin{array}{l}\text { Christine Hartmann } \\
\text { USA }\end{array}$ \\
\hline $\begin{array}{l}\text { Martin Gulliford } \\
\text { UK }\end{array}$ & $\begin{array}{l}\text { Antje Hammer } \\
\text { Germany }\end{array}$ & $\begin{array}{l}\text { Abraham Hartzema } \\
\text { USA }\end{array}$ \\
\hline $\begin{array}{l}\text { How-Ran Guo } \\
\text { Taiwan }\end{array}$ & $\begin{array}{l}\text { David Hanauer } \\
\text { USA }\end{array}$ & $\begin{array}{l}\text { Mohammed Harun-Or-Rashid } \\
\text { Japan }\end{array}$ \\
\hline $\begin{array}{l}\text { Indrani Gupta } \\
\text { India }\end{array}$ & $\begin{array}{l}\text { Amresh Hanchate } \\
\text { USA }\end{array}$ & $\begin{array}{l}\text { Carol Harvey } \\
\text { Australia }\end{array}$ \\
\hline $\begin{array}{l}\text { Birgitta } \AA \text { Gustafsson } \\
\text { Sweden }\end{array}$ & $\begin{array}{l}\text { Nicola Hancock } \\
\text { UK }\end{array}$ & $\begin{array}{l}\text { Gill Harvey } \\
\text { UK }\end{array}$ \\
\hline $\begin{array}{l}\text { Lars L Gustafsson } \\
\text { Sweden }\end{array}$ & $\begin{array}{l}\text { Rene Handschu } \\
\text { Germany }\end{array}$ & $\begin{array}{l}\text { M Hassanein } \\
\text { UK }\end{array}$ \\
\hline $\begin{array}{l}\text { Inaki Gutierrez } \\
\text { Spain }\end{array}$ & $\begin{array}{l}\text { Johanna Hanefeld } \\
\text { UK }\end{array}$ & $\begin{array}{l}\text { Henna Hasson } \\
\text { Sweden }\end{array}$ \\
\hline $\begin{array}{l}\text { Sally Guttmacher } \\
\text { USA }\end{array}$ & $\begin{array}{l}\text { Breffni Hannon } \\
\text { Canada }\end{array}$ & $\begin{array}{l}\text { Arvid Steinar Haugen } \\
\text { Norway }\end{array}$ \\
\hline $\begin{array}{l}\text { Astrid Guttmann } \\
\text { Canada }\end{array}$ & $\begin{array}{l}\text { Anne Helen Hansen } \\
\text { Norway }\end{array}$ & $\begin{array}{l}\text { Mona Haugum } \\
\text { Norway }\end{array}$ \\
\hline $\begin{array}{l}\text { Sudesh Gyawali } \\
\text { Nepal }\end{array}$ & $\begin{array}{l}\text { Bo Terning Hansen } \\
\text { Norway }\end{array}$ & $\begin{array}{l}\text { Leslie Hausmann } \\
\text { USA }\end{array}$ \\
\hline $\begin{array}{l}\text { Hanna Gyllensten } \\
\text { Sweden }\end{array}$ & $\begin{array}{l}\text { Kristian Hansen } \\
\text { UK }\end{array}$ & $\begin{array}{l}\text { Rachel Havyer } \\
\text { USA }\end{array}$ \\
\hline $\begin{array}{l}\text { Yossi Hadad } \\
\text { Israel }\end{array}$ & $\begin{array}{l}\text { Louise Hansen } \\
\text { Denmark }\end{array}$ & $\begin{array}{l}\text { Nikki Hawkins } \\
\text { USA }\end{array}$ \\
\hline $\begin{array}{l}\text { Anna Häger Glenngård } \\
\text { Sweden }\end{array}$ & $\begin{array}{l}\text { Claudia Hanson } \\
\text { Sweden }\end{array}$ & $\begin{array}{l}\text { Mark Hawley } \\
\text { UK }\end{array}$ \\
\hline $\begin{array}{l}\text { Anna Bettina Haidich } \\
\text { Greece }\end{array}$ & $\begin{array}{l}\text { Piya Hanvoravongchai } \\
\text { Thailand }\end{array}$ & $\begin{array}{l}\text { Alan Haycox } \\
\text { UK }\end{array}$ \\
\hline $\begin{array}{l}\text { Peter Hajek } \\
\text { UK }\end{array}$ & $\begin{array}{l}\text { Yanhua Hao } \\
\text { China }\end{array}$ & $\begin{array}{l}\text { Qusay Haydour } \\
\text { USA }\end{array}$ \\
\hline $\begin{array}{l}\text { Mark Hall } \\
\text { USA }\end{array}$ & $\begin{array}{l}\text { Patrick Hardigan } \\
\text { USA }\end{array}$ & $\begin{array}{l}\text { Philip Haywood } \\
\text { Australia }\end{array}$ \\
\hline $\begin{array}{l}\text { Dagmar Haller } \\
\text { Switzerland }\end{array}$ & $\begin{array}{l}\text { Jochen Hardt } \\
\text { Germany }\end{array}$ & $\begin{array}{l}\text { Na He } \\
\text { China }\end{array}$ \\
\hline $\begin{array}{l}\text { Pinchas Halpern } \\
\text { Israel }\end{array}$ & $\begin{array}{l}\text { Melanie Harling } \\
\text { Germany }\end{array}$ & $\begin{array}{l}\text { Larry Hearld } \\
\text { USA }\end{array}$ \\
\hline
\end{tabular}


Marilynne Hebert

Canada

Oskari Heikinheimo

Finland

Michele Heisler

USA

Ramzi Helewa

Canada

Karla Hemesath

USA

Jane Henderson

UK

Michelle Hendriks

Netherlands

Kène Henkens

Netherlands

Peter Herbison

New Zealand

Abraham Herbst

South Africa

Pauline Heslop

UK

Gijs Hesselink

Netherlands

Suzanne Heurtin-Roberts

USA

Sarah Hewlett

UK

Sharon Hewner

USA

Peter Heywood

Australia

Judith Hibbard

USA

Irene J Higginson

UK

Sue Hignett

UK

Helmut Hildebrandt

Germany

Mickael Hiligsmann

Netherlands

Peter Hill

Australia
Janet Hiller

Australia

Susan Hillier

Australia

Peter Hindley

UK

David Hipgrave

Australia

Lisa Hirschhorn

USA

Karen Hirschman

USA

Jennifer Hirshfeld-Cytron

USA

Jane Hirst

UK

Helena Hnilicová

Czech Republic

Vivian Ho

USA

Matthias Hoben

Canada

Matthias Hochadel

Germany

Marilyn Hodgins

Canada

Erik Hoencamp

Netherlands

Alexander Hoerbst

Austria

Thomas Hoerger

USA

Aubri Hoffman

USA

Barbara Hoffmann

Germany

Tammy Hoffmann

Australia

Michael Höfler

Germany

Hans Högberg

Sweden

Hans V Hogerzeil

Netherlands
Hans Hogerzeil

Switzerland

Petra Högger

Germany

Christa Hojlo

USA

Monika Hollander

Netherlands

Kathleen Holloway

Switzerland

Lewis B. Holmes

USA

Caroline Homer

Australia

Jin Pyo Hong

South Korea

Danielle Hoover

USA

Ann Hopton

UK

Monjurul Hoque

South Africa

Frances Horgan

Ireland

Klaus Hornetz

Germany

Simon Horsburgh

New Zealand

Krishna Hort

Australia

Salih Hosoglu

Turkey

David Hotchkiss

USA

Kreshnik Hoti

Australia

Allan House

UK

Tami Howe

New Zealand

Susan Hrisos

UK

An-Fu Hsiao

USA 
Hui-Min Hsieh

Taiwan

Ya-Seng Hsueh

Australia

Shanlian $\mathrm{Hu}$

China

Yanping Huang

Hong Kong

Sean Shenghsiu Huang

USA

\section{Kun Huang}

China

Marco Huesch

USA

David Hui

USA

Robbert Huijsman

Netherlands

Mark Hull

Canada

Marjan Hummel

Netherlands

Debbie Humphries

USA

Benjamin Hunter

UK

Rachael Hunter

UK

Krista Huot

USA

Catherine Hurley

Australia

Samia Hurst

Switzerland

Raymond Hutubessy

Switzerland

Ellen Idler

USA

Francesca Ieva

Italy

Friedrich Ihler

Germany

Shunya Ikeda

Japan
David Ikkersheim

Netherlands

Zubairu Iliyasu

Nigeria

Yuichi Imanaka

Japan

Devon Indig

Australia

Tor Ingebrigtsen

Norway

Richard Ingram

USA

David Inwald

UK

Uma M Irfan

Pakistan

Tadashi Ishida

Japan

M. Mofizul Islam

Australia

Leyla Ismayilova

USA

Hiroto Ito

Japan

Kouta Ito

USA

Hilde Hestad Iversen

Norway

Valentina Cabral Iversen

Norway

Chimaraoke Izugbara

Nigeria

Christian Olaf Jacke

Germany

Claire Jackson

Australia

Debra Jackson

South Africa

Diana Jackson

UK

Susan Jaglal

Canada

Albrecht Jahn

Germany
Gro Jamtvedt

Norway

Monika Janda

Australia

Heidi Janssen

Australia

Lina Jaruseviciene

Lithuania

Sara Javanparast

Australia

Masood Jawaid

Pakistan

Anuradha Jayanti

UK

Anupam Jena

USA

Natasja Koitzsch Jensen

Denmark

Marija Jevtic

Serbia

Huanguang Jia

USA

Liu Jianxun

China

Masahito Jimbo

USA

Chenggang Jin

China

Huajie Jin

UK

Mark R D Johnson

UK

Peter Johnson

USA

Bridget Johnston

UK

Jukka Jokinen

Finland

Carys Jones

UK

Lorelei Jones

UK

Ray Jones

UK 
Grace Joshy

Australia

Aleksandar Jovanovic

Serbia

Noel Juban

Philippines

Gyuchan Thomas Jun

UK

Ulrike Junius-Walker

Germany

Mary Justin-Temu

Tanzania

Haytham Kaafarani

USA

Axel Kaehne

UK

David Kaelber

USA

Natan Kahan

Israel

Ichiro Kai

Japan

Daphne Kaitelidou

Greece

Dan Kajungu

Uganda

László Kalabay

Hungary

Minal Kale

USA

Soumik Kalita

India

Lisa Kalman

USA

Lalit Kalra

UK

Eva Kaltenthaler

UK

Mary Kamb

USA

Taro Kamigaki

Japan

Abir Kanaan

USA
Donghun Kang

South Korea

Tipaporn Kanjanarach

Thailand

Hyacinthe Kankeu Tchewonpi

France

Lucy Kanya

Kenya

Dharmi Kapadia

UK

Anup Karan

India

Rajendra Karkee

Nepal

Jon Karnon

Australia

Monika Kastner

Canada

Gohei Kato

Japan

Noriko Kato

Japan

Mary Kawonga

South Africa

Kassoum Kayentao

Mali

Nicola Kayes

New Zealand

Peter Kazembe

Malawi

Benjamin Kearns

UK

Guy Kegels

Belgium

Margaret Kelaher

Australia

Anita Keller

Switzerland

Mary Lou Kelley

Canada

Anne Kennedy

UK

Patricia Kenny

Australia
Paula Kersten

UK

Stefan Kertesz

USA

Andrew Kestler

Canada

Jade Khalife

Lebanon

Jahangir Am Khan

Bangladesh

Pornsak Khortwong

Thailand

Juliet Kiguli

Uganda

Reinhold Kilian

Germany

Sue Kilpatrick

Australia

Sun-Young Kim

USA

James Kimani

Kenya

Shauna Kingsnorth

Canada

Russell Kirby

USA

Doris Kirigia

Kenya

Joses Muthuri Kirigia

Congo

Stephan Kirschner

Germany

Roman Kislov

UK

Alison Kitson

Australia

Suzanne Kiwanuka

Uganda

Lars Kjellin

Sweden

Egil Kjerstad

Norway

Caprice Knapp

USA 
Herschel Knapp
USA

Martin Knapp

UK

Andrew Knighton

USA

Jennifer Knopp-Sihota

Canada

Naonori Kodate

Ireland

Leonardo Koeser

UK

Gerjo Kok

Netherlands

Maryse Kok

Netherlands

Michaela Kolbe

Switzerland

Daniela Koller

Germany

Masahide Kondo

Japan

Alice Kongsted

Denmark

Alexander Konnopka

Germany

Antonis Kontemeniotis

Cyprus

Evangelos Kontopantelis

UK

Sascha Köpke

Germany

Holly Korda

USA

Deborah Korenstein

USA

Thomas Kötter

Germany

Anita Kotwani

India

Camille Eric Kouam

Canada

Paul Kowal

Switzerland
Markus Kraus

Austria

Anand Krishnan

India

Solvejg Kristensen

Denmark

Troels Kristensen

Denmark

Jimmie Kristensson

Sweden

Kurt Kroenke

USA

Margaret Elizabeth Kruk

USA

Silvia Krumm

Germany

Elizabeth Krupinski

USA

Jeffrey Kullgren

USA

Chandan Kumar

India

B Nirmal Kumar

UK

Saravana Kumar

Australia

Vishwajeet Kumar

India

Abera Kumie

Ethiopia

Ludwig Kuntz

Germany

Cynthia Kurtz

USA

Talma Kushnir

Israel

Bethany Kwan

USA

Wang Kwua-Yun

Taiwan

Ilias-Ioannis Kyriopoulos

Greece

Ulrich Laaser

Germany
Tracey-Lea Laba

Australia

Laishram Ladusingh

India

Martine Lagace

Canada

Chandrakant Lahariya

India

Claas Lahmann

Germany

Maria Lahuerta

USA

Christiaan Lako

Netherlands

Héctor Lamadrid-Figueroa

Mexico

Stephen Lambert

Australia

Majda Lamkaddem

Netherlands

Bruce Landon

USA

Peter Lange

Denmark

Claudia Langebrake

Germany

Maaike Langelaan

Netherlands

Peter Larson

USA

Bruce Larson

USA

Francis Lau

Canada

Rosa Lau

UK

Michael Lauerer

Germany

Julie Lauffenburger

USA

Anthony Laverty

UK

Dana Lawrence

USA 
Jeffrey Lazarus

Denmark

Amanda Leach

Australia

Jenine Leal

Canada

Andrew Chee Keng Lee

UK

Crystal Lee

Australia

Jae-Ho Lee

South Korea

Yu-Chen Lee

UK

Miaw-Chwen Lee

Taiwan

Mariska M G Leeflang

Netherlands

Jennifer Leeman

USA

Mengistu Legesse

Ethiopia

Sandra Leggat

Australia

David Legge

Australia

Beverly Leipert

Canada

Michael Leiter

Canada

Margus Lember

Estonia

Chryssoula Lemonidou

Greece

Kenneth L Leonard

USA

Aaron Leppin

USA

France Lert

France

Myles Leslie

USA

Ky Leung

Hong Kong
Ann Levin

USA

Carol Levin

USA

Robert Levine

USA

Terry Lewin

Australia

George Lewith

UK

Rui Li

USA

Gui Li

China

Ian $\mathrm{Li}$

Australia

Guohong Li

China

Xiaohong Li

China

Yuyuan Li

China

Zhijian Li

China

Yi-Ran Li

China

Xiaoyan Li

USA

Ya-Hsin Li

Taiwan

Min Lian

USA

Ying Liang

China

Hsienming Lien

Taiwan

Margareta Lilja

Sweden

Rebbecca Lilley

New Zealand

Wen Kwang Lim

Australia

Teck-Onn Lim

Malaysia
Rosemary Lim

UK

Chulaporn Limwattananon

Thailand

Hualiang Lin

China

Chun-Pin Lin

Taiwan

Wender Lin

Taiwan

Wolfgang Linden

Canada

Helena Lindgren

Sweden

Tove Lindhardr

Denmark

Martin Lindstrom

Sweden

Matteo Lippi Bruni

Italy

Martin Lipsky

USA

George Liu

Australia

Hongjie Liu

USA

Hong Liu

China

Jinfen Liu

China

Songsong Liu

UK

Su Liu

Hong Kong

Xiaoyun Liu

China

Lucylynn Lizarondo

Australia

Peter Lloyd-Sherlock

UK

Carl Lombard

South Africa

Janet Long

Australia 
Kim Longfield

USA

Yves Longtin

Canada

Luciane Cruz Lopes

Brazil

Fabiana Lorencatto

UK

Paula Lorgelly

Australia

Svetla Loukanova

Germany

Lesley Lowes

UK

Sam Lubner

USA

Tim Luckett

Australia

Wiebke Ludwig-Peitsch

Germany

Chi-Wai Lui

Australia

Jorge Luna

USA

Iréne Lund

Sweden

Sheng Luo

USA

May Nawal Lutfiyya

USA

Paula Luz

Brazil

Imogen Lyons

UK

Theodore Lystig

USA

Wei Ma

China

Thusitha Mabotuwana

USA

Christine Macarthur

UK

Marjorie Macdonald

Canada
Scott Macdonald

Canada

Anne Macfarlane

Ireland

Jane Macha

Tanzania

Adrian Mackenzie

Canada

Timothy Mackey

USA

Stuart Macleod

Canada

Malcolm Maclure

Canada

Deepak Rajaram Madi

India

Kenneth Maes

USA

Andreas Maetzel

Canada

Yodi Mahendradhata

Indonesia

Richard Mahoney

South Korea

Jenson Mak

Australia

Keijo Mäkelä

Finland

Matti Mäkelä

Finland

Madeeha Malik

Pakistan

Robert Malkin

USA

Margit Malmmose

Denmark

Mats Malqvist

Sweden

Stephen Maluka

Tanzania

Toshie Manabe

Japan

Rosemary Mander

UK
Lindsay Mangham-Jefferies

UK

Fatuma Manzi

Tanzania

Javier Mar

Spain

Bruno Marchal

Belgium

Giulio Marchesini

Italy

David Margel

Israel

Silvio Paolo Mariotti

Switzerland

Maureen Markle-Reid

Canada

Tania Markovic

Australia

Elsa Marques

UK

Paul Marschall

Germany

Andrea Marshall

Australia

Ernie Marshall

UK

Patrick Marshall

UK

Graham Martin

UK

Adriane Martin Hilber

Switzerland

Audibert Martine

France

Silvia Martinez Valverde Mexico

Anne Martin-Matthews

Canada

Eliana Cristina Martins Miranda Brazil

Michael Marx

Germany

Kijakazi Mashoto

Tanzania 
Felix Masiye

Zambia

Caroline Masquillier

Belgium

Inke Mathauer

Switzerland

John D Mathews

Australia

Klazien Matter-Walstra

Switzerland

\section{Stefano Mattioli}

Italy

Marco Matucci-Cerinic

Italy

Massimo Maurici

Italy

Thandisizwe Redford Mavundla

South Africa

Carl May

UK

Serge Mayaka Ma-Nitu

Belgium

Jo Maybin

UK

Jose Mayordomo

Spain

Bongani Mawethu Mayosi

South Africa

Olena Mazurenko

USA

Pamela Mazzocato

Sweden

Patrick Mbindyo

Kenya

Nicholas Mc Innes

Italy

Kevin Mc Namara

Australia

Abigail Mcbirnie

UK

Gordon Mccord

USA

Ewan Mcdonald

Australia
Julie Mcdonald

Australia

Rosemary Mcginnes

Australia

Gerry Mcgivern

UK

Matthew Mcgrail

Australia

Cynthia Mcgrath

USA

Meredith Mcintyre

Australia

Suzanne Mckenzie

Australia

Andrew Mclachlan

Australia

Mary-Louise Mclaws

Australia

Susannah Mclean

UK

Christopher Mcleod

Canada

Monsey Mcleod

UK

Laurence Mcmahon

USA

Juliet Mcmullin

USA

Kelly Mcqueen

USA

Nicolas Meda Burkina

Faso

Lourdes Medina

Puerto Rico

Laurenz Meier

Switzerland

Sarah Meier

USA

Nadja Meisterhans

Germany

Cara Meixner

USA

Michelle Mello

USA
Shanthi Mendis

Switzerland

Matthew Menear

Canada

Qingyue Meng

China

Stephen Mennemeyer

USA

Emmanouil Mentzakis

UK

Nicolas Menzies

USA

Joseph Menzin

USA

Lisa Merry

Canada

Andrea Messori

Italy

Herman Meulemans

Belgium

Lisbet Meurling

Sweden

Thorsten Meyer

Germany

Gesine Meyer-Rath

South Africa

Bernhard Michalowsky

Germany

Kristien Michielsen

Belgium

Nicos Middleton

Cyprus

Evelinn Mikkelsen

Netherlands

Anneli Milen

Finland

Anton Miller

Canada

Edward Miller

USA

Raffi Miller

USA

Robert Miller

USA 
Peggy Millson

Canada

Milan Milosevic

Croatia

William Min

USA

Mirella Minkman

Netherlands

Karin Minnie

South Africa

Silvano Mior

Canada

Jose Joaquin Mira

Spain

Nicholas Mitsakakis

Canada

Mitsunori Miyashita

Japan

Paul Mkandawire

Canada

Blandina Theophil Mmbaga

Norway

Rikke Moe

Norway

Deepika Mohan

USA

Katia Mohindra

Canada

David Mohr

USA

Mohammed Mohsin

Australia

Andrew Molodynski

UK

Shin-Ichi Momomura

Japan

Floriana Monciotti

Italy

Karen Monsen

USA

Ali Montazeri

Iran

Jesus Montero-Marin

Spain
Anthony Montgomery

Greece

Ann Moore

USA

David Moore

Canada

Jennifer Moore

New Zealand

Lynne Moore

Canada

Rachael Moorin

Australia

Mwifadhi Mrisho

Tanzania

Ashar Muhammad Malik

Pakistan

Axel Mühlbacher

Germany

Oscar J. Mujica

USA

Susmita Mukhopadhyay

India

Caroline Mulvaney

UK

Zubia Mumtaz

Canada

Kenneth Munge

Kenya

Fiona Munro

UK

Tariq Munshi

Canada

Mohammad Hassan Murad

USA

Adrianna Murphy

UK

Elizabeth Murray

UK

Jenni Murray

UK

Nandita Murukutla India

Wilbroad Mutale

Zambia
David Muthaka

Kenya

Conrad Muzoora

Uganda

Corrie Myburgh

Denmark

Dries Myny

Belgium

Julie Mytton

UK

Juliet Nabyonga Orem

Uganda

Matthias Nachtnebel

Australia

Farooq Naeem

Pakistan

Kent Nakamoto

USA

Toshimi Nakanishi Japan

Ireen Namakhoma

Malawi

Susan Nancarrow

Australia

Jagdeep Nanchahal

UK

Takashi Naruse

Japan

Rose Nathan

Tanzania

Corina Naughton

UK

Mwidimi Ndosi

UK

Sume Ndumbe-Eyoh

Canada

Graham Neilsen

Australia

Maria Ines B Nemes

Brazil

Sandeep S Nerkar

Sweden

Jonathan Neufeld

USA 


\begin{tabular}{|c|c|c|}
\hline Duncan Neuhauser & Dino Numerato & Elizabeth Oloruntoba \\
\hline USA & UK & Nigeria \\
\hline Josh Neumiller & Roberto Nuño-Solinís & Samuel Olowookere \\
\hline USA & Spain & Nigeria \\
\hline William Newbrander & Robin Nwankwo & Lars-Eric Olsson \\
\hline USA & USA & Sweden \\
\hline Phung Anh Nguyen & Francis Obare & Femi Omole \\
\hline Taiwan & Kenya & South Africa \\
\hline Ha Nguyen & Jon Oyvind Odland & Joseph Onakewhor \\
\hline Australia & Norway & Nigeria \\
\hline Thinh Nguyen & Brian Odonoghue & Ciaran O'Neill \\
\hline Australia & Australia & Ireland \\
\hline Pham Nguyen Bang & Olumuyiwa Odusanya & Jason Ong \\
\hline Vietnam & Nigeria & Australia \\
\hline Michael B Nichol & Pia Oedewald & Pierre Ongolo-Zogo \\
\hline USA & Finland & Cameroon \\
\hline Paul Nicholson & Ju Lee Oei & Charles Opondo \\
\hline UK & Australia & Kenya \\
\hline Nicola Nicolotti & Uzor Ogbu & Dermot O'Reilly \\
\hline Italy & USA & UK \\
\hline Jason Xin Nie & Russel Ogden & Aaron Orkin \\
\hline Canada & Canada & Canada \\
\hline Jens-Uwe Niehoff & Tinuade Ogunlesi & Carljohan Orre \\
\hline Germany & Nigeria & Sweden \\
\hline Signe Smith Nielsen & In-Hwan Oh & Jacques Orvain \\
\hline Denmark & South Korea & France \\
\hline Barnabas Njozing & Jude Uzoma Ohaeri & Dominik Ose \\
\hline Kenya & Nigeria & Germany \\
\hline Edward Nketiah-Amponsah & Bernadette O'Hare & Helmut Ostermann \\
\hline Ghana & Malawi & Germany \\
\hline Godswill Nnaji & Elialilia S Okello & Herwig Ostermann \\
\hline Nigeria & Uganda & Austria \\
\hline Lucia Nobilio & Christian Okolo & Laysha Ostrow \\
\hline Italy & Nigeria & USA \\
\hline Natasha Noble & Oladepo Oladimeji & Ronan O'Sullivan \\
\hline Australia & Nigeria & Ireland \\
\hline Haruko Noguchi & Joyce Olenja & Martin Ota \\
\hline Japan & Kenya & Congo \\
\hline Susan L Norris & John Oliffe & Angel Otero \\
\hline USA & Canada & Spain \\
\hline Shane Norris & Juan Oliva-Moreno & Wilma Otten \\
\hline South Africa & Spain & Netherlands \\
\hline Nicola North & Kathryn Oliver & Lixin Ou \\
\hline New Zealand & UK & Australia \\
\hline
\end{tabular}




\begin{tabular}{|c|c|c|}
\hline $\begin{array}{l}\text { Jan Oyebode } \\
\text { UK }\end{array}$ & $\begin{array}{l}\text { Gareth Parry } \\
\text { USA }\end{array}$ & $\begin{array}{l}\text { Virtudes Perez-Jover } \\
\text { Spain }\end{array}$ \\
\hline $\begin{array}{l}\text { Doruk Ozgediz } \\
\text { USA }\end{array}$ & $\begin{array}{l}\text { Matthew Parsons } \\
\text { New Zealand }\end{array}$ & $\begin{array}{l}\text { David Perkins } \\
\text { Australia }\end{array}$ \\
\hline $\begin{array}{l}\text { Jamie Padmore } \\
\text { USA }\end{array}$ & $\begin{array}{l}\text { Helen Parsons } \\
\text { USA }\end{array}$ & $\begin{array}{l}\text { John Perry } \\
\text { Canada }\end{array}$ \\
\hline $\begin{array}{l}\text { Andrew Page } \\
\text { Australia }\end{array}$ & $\begin{array}{l}\text { Minal Patel } \\
\text { USA }\end{array}$ & $\begin{array}{l}\text { Stephen Persell } \\
\text { USA }\end{array}$ \\
\hline $\begin{array}{l}\text { Kathleen Page } \\
\text { USA }\end{array}$ & $\begin{array}{l}\text { Preeti Patel } \\
\text { UK }\end{array}$ & $\begin{array}{l}\text { Gregory Peterson } \\
\text { Australia }\end{array}$ \\
\hline $\begin{array}{l}\text { Charles John Palenik } \\
\text { USA }\end{array}$ & $\begin{array}{l}\text { Andrea Patey } \\
\text { Canada }\end{array}$ & $\begin{array}{l}\text { Stefan Peterson } \\
\text { Sweden }\end{array}$ \\
\hline $\begin{array}{l}\text { Luigi Palestini } \\
\text { Italy }\end{array}$ & $\begin{array}{l}\text { Elisabetta Patorno } \\
\text { USA }\end{array}$ & $\begin{array}{l}\text { Anastas Philalithis } \\
\text { Greece }\end{array}$ \\
\hline $\begin{array}{l}\text { Lawrence Palinkas } \\
\text { USA }\end{array}$ & $\begin{array}{l}\text { Sue Pavitt } \\
\text { UK }\end{array}$ & $\begin{array}{l}\text { Mit Philips } \\
\text { Belgium }\end{array}$ \\
\hline $\begin{array}{l}\text { Laura Palombi } \\
\text { USA }\end{array}$ & $\begin{array}{l}\text { Ioanna Pavlopoulou } \\
\text { Greece }\end{array}$ & $\begin{array}{l}\text { Ceri Phillips } \\
\text { UK }\end{array}$ \\
\hline $\begin{array}{l}\text { Pradeep Panda } \\
\text { India }\end{array}$ & $\begin{array}{l}\text { Milena Pavlova } \\
\text { Netherlands }\end{array}$ & $\begin{array}{l}\text { James Phillips } \\
\text { USA }\end{array}$ \\
\hline $\begin{array}{l}\text { Massimiliano Panella } \\
\text { Italy }\end{array}$ & $\begin{array}{l}\text { Manjiri Pawaskar } \\
\text { USA }\end{array}$ & $\begin{array}{l}\text { Lawrence Phillips } \\
\text { UK }\end{array}$ \\
\hline $\begin{array}{l}\text { Supasit Pannarunothai } \\
\text { Thailand }\end{array}$ & $\begin{array}{l}\text { Christopher Pearce } \\
\text { Australia }\end{array}$ & $\begin{array}{l}\text { Lyn Phillipson } \\
\text { Australia }\end{array}$ \\
\hline $\begin{array}{l}\text { George Papachristou } \\
\text { Greece }\end{array}$ & $\begin{array}{l}\text { Rupert Pearse } \\
\text { UK }\end{array}$ & $\begin{array}{l}\text { Fiammetta Piersigilli } \\
\text { Italy }\end{array}$ \\
\hline $\begin{array}{l}\text { Evelina Pappa } \\
\text { Greece }\end{array}$ & $\begin{array}{l}\text { Nancye May Peel } \\
\text { Australia }\end{array}$ & $\begin{array}{l}\text { Arwen Pieterse } \\
\text { Netherlands }\end{array}$ \\
\hline $\begin{array}{l}\text { Hong-Jae Park } \\
\text { New Zealand }\end{array}$ & $\begin{array}{l}\text { Jose Peeters } \\
\text { Netherlands }\end{array}$ & $\begin{array}{l}\text { Clément Pimouguet } \\
\text { France }\end{array}$ \\
\hline $\begin{array}{l}\text { Young Sik Park } \\
\text { South Korea }\end{array}$ & $\begin{array}{l}\text { Salvador Peiró } \\
\text { Spain }\end{array}$ & $\begin{array}{l}\text { Hilary Pinnock } \\
\text { UK }\end{array}$ \\
\hline $\begin{array}{l}\text { Sophie Park } \\
\text { UK }\end{array}$ & $\begin{array}{l}\text { Ashleigh Pell } \\
\text { UK }\end{array}$ & $\begin{array}{l}\text { Aarão Mendes Pinto Neto } \\
\text { Brazil }\end{array}$ \\
\hline $\begin{array}{l}\text { Louise Parker } \\
\text { USA }\end{array}$ & $\begin{array}{l}\text { James Peltier } \\
\text { USA }\end{array}$ & $\begin{array}{l}\text { Jorge Pinzon } \\
\text { Canada }\end{array}$ \\
\hline $\begin{array}{l}\text { Vicki Parker } \\
\text { Australia }\end{array}$ & $\begin{array}{l}\text { Mark Pennington } \\
\text { UK }\end{array}$ & $\begin{array}{l}\text { Stephen Pitts } \\
\text { USA }\end{array}$ \\
\hline $\begin{array}{l}\text { Aimee Parnell } \\
\text { USA }\end{array}$ & $\begin{array}{l}\text { Beata Peplonska } \\
\text { Poland }\end{array}$ & $\begin{array}{l}\text { Anne Marie Plass } \\
\text { Netherlands }\end{array}$ \\
\hline $\begin{array}{l}\text { Adriana Parrella } \\
\text { Australia }\end{array}$ & $\begin{array}{l}\text { Jose Pereira } \\
\text { Canada }\end{array}$ & $\begin{array}{l}\text { Christian Plessen } \\
\text { Denmark }\end{array}$ \\
\hline $\begin{array}{l}\text { David Parry } \\
\text { New Zealand }\end{array}$ & $\begin{array}{l}\text { Ricardo Perez-Cuevas } \\
\text { Mexico }\end{array}$ & $\begin{array}{l}\text { Thomas Plochg } \\
\text { Netherlands }\end{array}$ \\
\hline
\end{tabular}


Ronald Plotnikoff

Australia

Rhys Pockett

UK

Dan Poenaru

Canada

Subhash Pokhrel

UK

Jeannette Pols

Netherlands

Marie-Pascale Pomey

Canada

Catherine Pope

UK

Brenten Popiel

USA

Aron Frederik Popov

Germany

Andrea Poscia

Italy

Douwe Postmus

Netherlands

Otto Melchior Poulsen

Denmark

Koen B. Pouwels

Netherlands

Colin Powell

UK

Timothy Powell-Jackson

UK

Alexis Pozen

USA

Wasana Prasitsuebsai

Thailand

Devarsetty Praveen

India

George Praygod

Tanzania

Dawn Prentice

Canada

Panagiotis Prezerakos

Greece

Mirela Prgomet

Australia
Morgan Price

Canada

Kathleen Pritchard

Canada

Ari Probandari

Indonesia

Birgit Prodinger

Switzerland

Ana Progovac

USA

Supannee Promthet

Thailand

Wendy Prudhomme O'Meara

Kenya

Dimitri Prybylski

USA

Jacqueline Pugh

USA

Sue Pullon

New Zealand

Rebecca Purc-Stephenson

Canada

Sampsa Puttonen

Finland

Peter Pype

Belgium

Feng Qian

USA

Xuezheng Qin

China

Troy Quast

USA

Wilm Quentin

Germany

Sally Quilligan

UK

Julie Quinlivan

Australia

Atonu Rabbani

Bangladesh

Fauziah Rabbani

Pakistan

Giovanni Rabito

Switzerland
Beth Rachlis

Canada

Rajiv Radhakrishnan

USA

Alberto Raggi

Italy

Lisa Rahangdale

USA

Anna Rahman

USA

Peter Raich

USA

Risto Raivio

Finland

Vahid Rakhshan

Iran

Mark Ralston

USA

Bram Ramaekers

Netherlands

Peter Rambau

Tanzania

Edmond Ramly

USA

Sue Randall

Australia

Gurprit Randhawa

Canada

Erik Ranschaert

Belgium

Jonas Ranstam

Sweden

Tapio Rantanen

Finland

Lars Rasmussen

Denmark

Freya Rasschaert

Belgium

Nithima Ratanasit

Thailand

Mihi Ratima

New Zealand

Ramin Ravangard

Iraq 
John Read

UK

Bernd Rechel

UK

Siddharta Reddy

USA

J A Reekers

Netherlands

Jemma Regan

UK

Krishna Regmi

UK

Jaroslaw Regula

Poland

Colin Reid

Canada

Stephen Reid

South Africa

Stewart Reid

Zambia

Thomas Reinhold

Germany

William Renwick

Australia

James Reschovsky

USA

Serge Resnikoff

Switzerland

Joseph Restuccia

USA

Kelly Reveles

USA

Debra Revere

USA

Heidi Reynolds

USA

Joanna Reynolds

UK

Abbas Rezaianzadeh

Iran

Reza Rezayatmand

Netherlands

Todd Rice

USA
Rebecca Richards-Kortum

USA

Solina Richter

Canada

Wietske Rienstra

Netherlands

Michael Rigby

Ireland

Ian Ring

Australia

Marit By Rise

Norway

Gilbert Ritschard

Switzerland

Kerstin Roback

Sweden

Adam Roberts

UK

Drucilla Roberts

USA

Jackie Robinson

New Zealand

Jaime Robledo

Colombia

Monica Robotin

Australia

Dylan Roby

USA

Peter Rockers

USA

Rosa Rodriguez-Monguio

USA

Cecilie Roe

Norway

Morwenna Rogers

UK

James Rohrer

USA

Martin Roland

UK

Sarah Rominski

USA

Janetta Roos

South Africa
Susan Rose

USA

Laura Rosella

Canada

Michael Rosen

USA

Pauline Rosenau

USA

Tom Ross

USA

Andrea Rotolo

Italy

Ronen Rozenblum

USA

Juan Ruano

Spain

Leonard Rubenstein

USA

Daniel Rubin

USA

Maria Rubio-Valera

Spain

Gavin Rudge

UK

Robert Rudin

USA

Claudia Rudroff

Germany

Peter Ruesch

Switzerland

Myriam Ruiz-Rodriguez

Colombia

Giuliano Russo

Portugal

Elizeus Rutebemberwa

Uganda

Geert Rutten

Netherlands

Joanne Ryan

Australia

Carla Sabariego

Germany

Lindsay Sabik

USA 


\begin{tabular}{|c|c|c|}
\hline $\begin{array}{l}\text { Mohsen Sadatsafavi } \\
\text { Canada }\end{array}$ & $\begin{array}{l}\text { Milena Sant } \\
\text { Italy }\end{array}$ & $\begin{array}{l}\text { Isabelle Scholl } \\
\text { Germany }\end{array}$ \\
\hline $\begin{array}{l}\text { Debasish Saha } \\
\text { Belgium }\end{array}$ & $\begin{array}{l}\text { Malay Sarkar } \\
\text { India }\end{array}$ & $\begin{array}{l}\text { Christoph Scholz } \\
\text { Germany }\end{array}$ \\
\hline $\begin{array}{l}\text { Olivier Saint-Lary } \\
\text { France }\end{array}$ & $\begin{array}{l}\text { Malabika Sarker } \\
\text { Bangladesh }\end{array}$ & $\begin{array}{l}\text { Miranda Schram } \\
\text { Netherlands }\end{array}$ \\
\hline $\begin{array}{l}\text { Adnan Saithna } \\
\text { UK }\end{array}$ & $\begin{array}{l}\text { Faruk Sarkinfada } \\
\text { Nigeria }\end{array}$ & $\begin{array}{l}\text { Jonas Schreyögg } \\
\text { Germany }\end{array}$ \\
\hline $\begin{array}{l}\text { Priyanka Saksena } \\
\text { Switzerland }\end{array}$ & $\begin{array}{l}\text { Marco Sartirana } \\
\text { Italy }\end{array}$ & $\begin{array}{l}\text { Paul Schultz } \\
\text { USA }\end{array}$ \\
\hline $\begin{array}{l}\text { Rehana Salam } \\
\text { Pakistan }\end{array}$ & $\begin{array}{l}\text { Sumathi Sathya } \\
\text { India }\end{array}$ & $\begin{array}{l}\text { Richard Schulz } \\
\text { USA }\end{array}$ \\
\hline $\begin{array}{l}\text { Abdus Salam } \\
\text { Malaysia }\end{array}$ & $\begin{array}{l}\text { Azusa Sato } \\
\text { UK }\end{array}$ & $\begin{array}{l}\text { Nadine Schuurman } \\
\text { Canada }\end{array}$ \\
\hline $\begin{array}{l}\text { Ellinor Salander Renberg } \\
\text { Sweden }\end{array}$ & $\begin{array}{l}\text { Mikiya Sato } \\
\text { Japan }\end{array}$ & $\begin{array}{l}\text { Hilary Schwandt } \\
\text { USA }\end{array}$ \\
\hline $\begin{array}{l}\text { Guillermo Salinas-Escudero } \\
\text { Mexico }\end{array}$ & $\begin{array}{l}\text { Stefan Sauerland } \\
\text { Germany }\end{array}$ & $\begin{array}{l}\text { Rene Schwendimann } \\
\text { Switzerland }\end{array}$ \\
\hline $\begin{array}{l}\text { Miguel Salinero-Fort } \\
\text { Spain }\end{array}$ & $\begin{array}{l}\text { Saw Saw Saw } \\
\text { Myanmar }\end{array}$ & $\begin{array}{l}\text { David Scrimgeour } \\
\text { Australia }\end{array}$ \\
\hline $\begin{array}{l}\text { Andrew Salner } \\
\text { USA }\end{array}$ & $\begin{array}{l}\text { Sylvia Sax } \\
\text { Germany }\end{array}$ & $\begin{array}{l}\text { David Seder } \\
\text { USA }\end{array}$ \\
\hline $\begin{array}{l}\text { Brendan Saloner } \\
\text { USA }\end{array}$ & $\begin{array}{l}\text { Shane Scahill } \\
\text { New Zealand }\end{array}$ & $\begin{array}{l}\text { Laurence Seematter-Bagnoud } \\
\text { Switzerland }\end{array}$ \\
\hline $\begin{array}{l}\text { Stacie Salsbury } \\
\text { USA }\end{array}$ & $\begin{array}{l}\text { Sasha Scambler } \\
\text { UK }\end{array}$ & $\begin{array}{l}\text { Joel Segel } \\
\text { USA }\end{array}$ \\
\hline $\begin{array}{l}\text { Katherine Salter } \\
\text { Canada }\end{array}$ & $\begin{array}{l}\text { Marco Scarpa } \\
\text { Italy }\end{array}$ & $\begin{array}{l}\text { Hanna Seidling } \\
\text { Germany }\end{array}$ \\
\hline $\begin{array}{l}\text { Domenico Salvatore } \\
\text { Italy }\end{array}$ & $\begin{array}{l}\text { Frederieke Schaafsma } \\
\text { Australia }\end{array}$ & $\begin{array}{l}\text { Polona Selic } \\
\text { Slovenia }\end{array}$ \\
\hline $\begin{array}{l}\text { Jane Sandall } \\
\text { UK }\end{array}$ & $\begin{array}{l}\text { Juergen Schaefer } \\
\text { Germany }\end{array}$ & $\begin{array}{l}\text { Sakthivel Selvaraj } \\
\text { India }\end{array}$ \\
\hline $\begin{array}{l}\text { Magnus Sandberg } \\
\text { Sweden }\end{array}$ & $\begin{array}{l}\text { Francois Schellevis } \\
\text { Netherlands }\end{array}$ & $\begin{array}{l}\text { Salaam Semaan } \\
\text { USA }\end{array}$ \\
\hline $\begin{array}{l}\text { Uwe Sander } \\
\text { Germany }\end{array}$ & $\begin{array}{l}\text { Jeffrey Scherrer } \\
\text { USA }\end{array}$ & $\begin{array}{l}\text { Catherine Sermet } \\
\text { France }\end{array}$ \\
\hline $\begin{array}{l}\text { Lars Sandman } \\
\text { Sweden }\end{array}$ & $\begin{array}{l}\text { Virginia Schmied } \\
\text { Australia }\end{array}$ & $\begin{array}{l}\text { Walter Sermeus } \\
\text { Belgium }\end{array}$ \\
\hline $\begin{array}{l}\text { Felipe Sandoval } \\
\text { Japan }\end{array}$ & $\begin{array}{l}\text { Helen Schneider } \\
\text { South Africa }\end{array}$ & $\begin{array}{l}\text { Tanya Seshadri } \\
\text { India }\end{array}$ \\
\hline $\begin{array}{l}\text { Gabriel Sanfélix-Gimeno } \\
\text { Spain }\end{array}$ & $\begin{array}{l}\text { Michael Schneider } \\
\text { USA }\end{array}$ & $\begin{array}{l}\text { Elizabeth Seston } \\
\text { UK }\end{array}$ \\
\hline $\begin{array}{l}\text { Claudia Sanmartin } \\
\text { Canada }\end{array}$ & $\begin{array}{l}\text { Veronika Schoeb } \\
\text { Switzerland }\end{array}$ & $\begin{array}{l}\text { Nick Sevdalis } \\
\text { UK }\end{array}$ \\
\hline
\end{tabular}


Melchior Seyfarth

Germany

Omid Shabestari

Canada

Baiju Shah

Canada

Madhusudana Shampur

India

Vandad Sharifi

Iran

Urvashi Sharma

UK

Adam Sharp

USA

Alison Shaw

UK

Charles Shaw

UK

Dorothy Shaw

Canada

L. Aubree Shay

USA

Ian Sheerin

New Zealand

Paul Shekelle

USA

Jay Shen

USA

Bryan Shepherd

USA

Yuyan Shi

USA

Shu-Fang Shih

Taiwan

Chih-Yuan Shih

Taiwan

Sophy Shih

Australia

Nathan Shippee

USA

Amir Shmueli

Israel

N Shorbaji

Switzerland
Camille Short

Australia

Zhou Shoujun

China

Mohsin Sidat

Mozambique

Grigory Sidorenkov

Netherlands

Mark Siedner

USA

Rüdiger Siekmeier

Germany

Volkert Siersma

Denmark

Jacques Silber

Israel

Jonathan Silcock

UK

Martha Silva

New Zealand

Leickness Chisamu Simbayi

South Africa

Douglas Simkiss

UK

Edina Sinanovic

South Africa

Timo Sinervo

Finland

Daniel Singer

USA

Sara Singer

USA

Simone Singh

USA

Dan Siskind

Australia

Eric Siskind

USA

Priyadharshini Sivakumaran Australia

Judith Sixsmith

UK

Steve Sizmur

UK
Ingeborg Strømseng Sjetne

Norway

Chris Skedgel

Canada

Kjersti Eeg Skudal

Norway

Susan Slade

Australia

Susan Slaughter

Canada

Nancy L Sloan

USA

Neil Smart

Australia

Cees Th. Smit Sibinga

Netherlands

Alison Smith

UK

Anthony Smith

Australia

Dean Smith

USA

Susan Smith

Ireland

Carolyn Smith-Morris

USA

Liz Smythe

New Zealand

Helene L Soberg

Norway

Paulina Sockolow

USA

Girish M Sogi

India

Mohammad Reza Sohrabi

Iran

Roy L. Soiza

UK

Maria Giuliana Solinas

Italy

Dominique Somme

France

David Sommerfeld

USA 
Benjamin Sommers

USA

Mark Sonderup

South Africa

Hummy Song

USA

Yunjie Song

USA

Werner Soors

Belgium

Elizaveta Sopina

Denmark

Jacob Thorsted Sorensen

Denmark

Jan Sorensen

Denmark

Atsushi Sorita

USA

Sandra G. Sosa-Rubi

Mexico

Kyriakos Souliotis

Greece

Paulo Sousa

Portugal

Mikle South

USA

Alex Sox-Harris

USA

Olaitan Soyannwo

Nigeria

Adedoyin Soyibo

Nigeria

Donat Rudolf Spahn

Switzerland

Lene Spanager

Denmark

Stuart Speedie

USA

Neil Spicer

UK

Mark Spigt

Netherlands

Anne Spinewine

Belgium
Andrew Springer

USA

Jayadevan Sreedharan

United Arab Emirates

Shalini Sri Ranganathan

Sri Lanka

Anurag Srivastava

India

Jeong Seok Sro

South Korea

Catherine St. Hill

USA

Cecilia Stålsby Lundborg

Sweden

Baudouin Standaert

Belgium

Hilary Standing

UK

Victoria Stanhope

USA

Susan Stapleton

USA

Laura Steinhardt

USA

Gregory Stevens

USA

Joanna Stewart

New Zealand

Jane Stewart

UK

Alessandro Stievano

Italy

Karyn Stitzenberg

USA

Jill Stocks

UK

Andrew Stoddart

UK

Johannes Stoelwinder

Australia

Emma Stokes

Ireland

Matthias Stoll

Germany
Jamie Stone

USA

Marianne Storm

Norway

Jackie Street

Australia

Matej Stuhec

Slovenia

Prasanth Subrahmanian

India

Dawn Sugarman

USA

Takehiro Sugiyama

Japan

Sarah Sullivan

UK

Sheena Sullivan

Australia

Elinor Sullivan

USA

Qi Sun

China

Reijo Sund

Finland

\section{Gerdt Sundstrom}

Sweden

Wayne Sunman

UK

Rosa Sunol

Spain

Tarja Suominen

Finland

Paibul Suriyawongpaisal

Thailand

György Surján

Hungary

Igor Svab

Slovenia

Elisabeth Svensson

Sweden

Dace Svikis

USA

Michael Swanoski

USA 
Deborah Swinglehurst

UK

Shabbir Syed-Abdul

Taiwan

Miriam Taegtmeyer

UK

Saeed Taheri

Iran

Hideto Takahashi

Japan

Hiromi Takahashi-Omoe

Japan

Zenobia Talati

Australia

Giorgio Tamburlini

Italy

Joseph Tan

Canada

Siok Swan Tan

Netherlands

Ryan Tandjung

Switzerland

Min Moon Tang

Malaysia

Shenglan Tang

USA

Wei Tang

Japan

Sripen Tantivess

Thailand

Tawesak Tanwandee

Thailand

Sihai Tao

China

Elizabeth Tarlov

USA

Sandra Tarquinio

Brazil

Robert Tate

Canada

Paula Tavrow

USA

Katja Taxis

Netherlands
Chelsea Taylor

Australia

Judy Taylor

Australia

Nicholas Taylor

Australia

Natalie Taylor

Australia

Sandra Taylor

USA

Katherine Taylor Haynes

USA

Gary Teare

Canada

Cristian Tebé

Spain

Marleen Temmerman

Belgium

Martha Ann Terry

USA

Angela Testi

Italy

Dilip Thandassery

India

Gudrun Thengisdottir

UK

Horst Thiermann

Germany

Jill Thistlethwaite

Australia

Aliki Thomas

Canada

Anne Thomas

USA

Bejoy Thomas

Canada

Roanne Thomas

Canada

Sandra Thompson

Australia

Ann Thomson

UK

William Murray Thomson

New Zealand
Richard Thomson

UK

Kevan Thorley

UK

Kamala Thriemer

Australia

Colin Thunhurst

UK

Lau Thygesen

Denmark

Stephanie Tierney

UK

Maike Tietschert

Netherlands

Stephen Timmons

UK

Samuel Tisherman

USA

Hale Toklu

USA

Krzysztof Tomaszewski

Poland

Sara Tomczyk

Ethiopia

Toshiyoshi Tominaga

Japan

Stephanie Topp

Zambia

Kwasi Torpey

Nigeria

Hidenori Toyoda

Japan

Valeria D. Tozzi

Italy

Joan Tranmer

Canada

Gerd Tranø

Norway

Jeanette Trauth

USA

Andrea Tricco

Canada

Ursula Trummer

Austria 
Jeroen Trybou

Belgium

Yafang Tsai

Taiwan

Jack Tsai

USA

Feng-Jen Tsai

Taiwan

Girmay Tsegay

Ethiopia

Apostolos Tsiachristas

Netherlands

Miyuki Tsuchihashi-Makaya

Japan

Hong Anh Tu

Netherlands

Carole Tucker

USA

Lars Tummers

Netherlands

Janet Turan

USA

Joanne Turnbull

UK

Janette Turner

UK

Sheila Turner

UK

Ingrid Tyler

Canada

Kerry Uebel

South Africa

Maduka Ughasoro

Nigeria

Nkolika Uguru

Nigeria

Jennifer Uhrig

USA

Waqar Ulhassan

Sweden

Antje Ullrich

Germany

Jane Umeano-Enemuoh

Nigeria
Hiroyuki Umegaki

Japan

Rosa M. Urbanos-Garrido

Spain

Christine Urquhart

UK

\section{Robin Urquhart}

Canada

Maritta Valimaki

Finland

Mentore Vaccari

Italy

Anil Vaidya

UK

Vivian Valdmanis

USA

Luke Vale

UK

Janet Van Cleave

USA

Kate Van De Vooren

Netherlands

Elske Van Den Akker

Netherlands

Patricia Van Den Bemt

Netherlands

Neeltje Van Den Berg

Germany

Hendrik Van Den Bussche

Germany

Jan Van Der Meulen

UK

Ineke Van Der Wulp

Netherlands

Willem Herbert Van Harten

Netherlands

Richard Van Kleef

Netherlands

Josefien Van Olmen

Belgium

Catharina Van Oostveen

Netherlands

Joost Van Veen

UK
Carol Vandeusen Lukas

USA

Per Olav Vandvik

Norway

Kris Vanhaecht

Belgium

Özalp Vayvay

Turkey

Nerina Vecchio

Australia

Alicia Vedio

UK

Pepijn Vemer

Netherlands

Bruno Ventelou

France

Rafael Vercelino

Brazil

R.A. Verheij

Netherlands

Arnoud Verhoeff

Netherlands

Marcia Vervloet

Netherlands

Reinhold Vieth

Canada

Stefano Villa

Italy

Deborah Vincent

USA

Katherine Virgo

USA

Susan Vlack

Australia

Lorenz Von Seidlein

Thailand

Thomas Vreugdenburg

Australia

Bert Vrijhoef

Singapore

Victoria Anne Wade

Australia

Norman Waitzman

USA 
Annika Waldmann

Germany

Agnes Walker

Australia

Kara Walker

USA

Amy Walker

USA

Emily Walkom

Australia

Emma Wallace

Ireland

John Walley

UK

Lars Wallin

Sweden

Bronagh Walsh

UK

Gill Walt

UK

Ulla Walter

Germany

Darren Walters

Australia

Stephen John Walters

UK

Edward Waltz

USA

Richard Wamai

USA

Nick Wan

USA

Anne Wand

Australia

Fahui Wang

USA

Guijing Wang

USA

Hufeng Wang

China

Hongmei Wang

China

Zhen Wang

USA
Jian Wang

China

Qing Wang

China

Ying Wang

China

Wei Wang

Australia

Christine Wann-Hansson

Sweden

Rhoda Wanyenze

Uganda

Jane Wardle

UK

Douglas Fraser Wares

Switzerland

Justin Waring

UK

Margaretha Warlé-Van Herwaarden

Netherlands

Charlotte Warren

Kenya

Narelle Warren

Australia

Steven Wartman

USA

Elizabeth Wasilevich

USA

Patrick Waterson

UK

Francois Watson

South Africa

Verity Watson

UK

Robert Wears

USA

William Weeks

USA

Li Wei

UK

Ji-Fu Wei

China

Xiaolin Wei

China
Matthias Weigl

Germany

John Wells

Ireland

Kristen Wells

USA

Susan Wells

New Zealand

Annalena Welp

Switzerland

Claus Wendt

Germany

Rhay-Hung Weng

Taiwan

Sophie Werkö

Sweden

Erik L. Werner

Norway

Colin West

USA

Laura Wherry

USA

Dean Whitehead

New Zealand

Stuart Whittaker

South Africa

David Whyatt

Australia

Siri Wiig

Norway

Heinrike Wilkens

Germany

Barbara Willey

UK

Anna Williams

Australia

Ann Williams

USA

Mark Williams

USA

Chad Williamson

USA

Sarah Willis

UK 
Douglas Wilson

South Africa

Khin Than Win

Australia

Haije Wind

Netherlands

Lori Wingate

USA

Brad Winters

USA

Meg Wise

USA

Jens Witsch

Germany

Barbara Wizner

Poland

Eileen Wllis

Australia

Priscilla Wobil

Ghana

Leslie Wolf

USA

Carlos King Ho Wong

Hong Kong

Edwin Wong

USA

Geoff Wong

UK

Sabrina Wong

Canada

Rachael Wood

UK

Fiona Wood

UK

Richard Woodman

Australia

Cynthia Woodsong

South Africa

Edwin Wouters

Belgium

Frances Wright

Canada

Bei Wu

USA
Lesley Wye

UK

Kaspar Wyss

Switzerland

Anping Xie

USA

Jian-Xiang Xie

China

Lingzhong $\mathrm{Xu}$

China

Ji-Jiang Yang

China

Li-Ye Yang

China

Yi-Hsin Yang

Taiwan

Philip Yanos

USA

Lorraine Yap

Australia

Marie Yap

Australia

Bobbi Jo Yarborough

USA

Aaron Yarlas

USA

Annalee Yassi

Canada

Barbara Yawn

USA

Bo Ye

UK

Xiaohua Ye

China

Wen Ye

USA

Peter Yellowlees

Australia

Laurann Yen

Australia

Hongmei Yi

China

Cheng-Har Yip

Malaysia
Jennifer Yip

UK

Candice Yong

USA

Hong Il Yoo

UK

Illhoi Yoo

USA

Jean Yoon

USA

Joyce You

Hong Kong

Tracey Young

UK

Sitaporn Youngkong

Thailand

Mustafa Younis

USA

Junhua Yu

USA

Beibei Yuan

Sweden

Zhaokang Yuan

China

Manwai Yuen

Hong Kong

Nicole Yurgin

USA

Mahmoud Zakherah

Egypt

Paola Zappa

Switzerland

Ehsan Zarei

Iran

Ruth Zaslansky

Germany

John Zeber

USA

Sten Zelle

Netherlands

Jennifer Zelmer

Canada

Wu Zeng

USA 
Floriana Zennaro

Italy

Isik Zeytinoglu

Canada

Xinping Zhang

China

Zhitao Zhang

China

Jingping Zhao

China
Kai Zheng

USA

Lei Zhou

China

Liang Zhou

China

Quan Zhou

China

Carolyn Zhu

USA
Junya Zhu

USA

Joseph Zickafoose

USA

Maria Zolfo

Belgium

Diana Zuckerman

USA

Yvonne Zurynski

Australia 